\title{
A unified NLO description of top-pair and associated Wt production
}

\author{
F. Cascioli ${ }^{\mathrm{a}}$, S. Kallweit ${ }^{\mathrm{b}}$, P. Maierhöfer ${ }^{\mathrm{c}}$, S. Pozzorini ${ }^{\mathrm{d}}$ \\ Institut für Theoretische Physik, Universität Zürich, 8057 Zurich, Switzerland
}

Received: 4 December 2013 / Accepted: 14 February 2014 / Published online: 4 March 2014

(c) The Author(s) 2014. This article is published with open access at Springerlink.com

\begin{abstract}
We present an NLO simulation of WWbb production with massive b-quarks at the LHC. Off-shell and non-resonant contributions associated with top-pair and single-top channels and with leptonic W-boson decays are consistently taken into account using the complex-mass scheme. Thanks to the finite b-quark mass, WWbb predictions can be extended to the whole b-quark phase space, thereby including Wt-channel single-top contributions that originate from collinear $\mathrm{g} \rightarrow$ bb splittings in the four-flavour scheme. This provides a consistent NLO description of tt and $\mathrm{Wt}$ production and decay, including quantum interference effects. The simulation is also applicable to exclusive 0 and 1-jet bins, which is of great importance for Higgs-boson studies in the $\mathrm{H} \rightarrow$ WW channel and for any other analysis with large top backgrounds and jet vetoes or jet bins.
\end{abstract}

\section{Introduction}

Top quarks are the heaviest known fundamental particles, and the precise theoretical understanding of their production and decay mechanism, within or beyond the Standard Model, has deep implications on countless aspects of the LHC physics programme. At the LHC, top quarks are mainly produced as $\mathrm{tt}^{\mathrm{t}}$ pairs and via single-top production in the $t$-channel or in the associated $\mathrm{Wt}$ mode. At $8 \mathrm{TeV}$ these latter single-top channels amount to 40 and $10 \%$ of the $\mathrm{t}^{\mathrm{t}}$ cross section, respectively. In spite of their smaller cross sections, they play an important role as direct probes of top-quark weak interactions and of their flavour structure. The separation of top-production into individual top-pair and single-top contributions poses non-trivial experimental and theoretical challenges, which are mainly due to the similarity among the final states asso-

\footnotetext{
a e-mail: cascioli@physik.uzh.ch

be-mail: kallweit@physik.uzh.ch

c e-mail: philipp@physik.uzh.ch

de-mail: pozzorin@physik.uzh.ch
}

ciated with the various mechanisms of top-production and decay. In particular, the definition of $\mathrm{t} \overline{\mathrm{t}}$ and $\mathrm{Wt}$ production involves notorious and quite subtle theoretical issues [1].

In the five-flavour $(5 \mathrm{~F})$ scheme, $\mathrm{Wt}$ production proceeds via b-quark induced partonic channels like $\mathrm{gb} \rightarrow \mathrm{W}^{-} \mathrm{W}^{+} \mathrm{b}$, and the presence of a single $b$-jet represents a clearly distinctive feature with respect to $\mathrm{W}^{+} \mathrm{W}^{-} \mathrm{b} \overline{\mathrm{b}}$ final states associated with tt production. However, beyond LO this separation ceases to exist, since $\mathrm{gg} \rightarrow \mathrm{W}^{+} \mathrm{W}^{-} \mathrm{b} \overline{\mathrm{b}}$ enters also the next-to-leading order (NLO) corrections to Wt production. The resulting $\mathrm{t} \overline{\mathrm{t}}$ contamination represents a huge NLO correction, which jeopardises the perturbative convergence of the $\mathrm{Wt}$ cross section in the $5 \mathrm{~F}$ scheme. To circumvent this problem within the $5 \mathrm{~F}$ scheme, various approaches have been proposed aimed at subtracting the contribution of a second top resonance in $\mathrm{pp} \rightarrow \mathrm{Wt}+X$ [1]. However, these prescriptions either break gauge invariance or are not applicable to a realistic experimental setup. Moreover they neglect the quantum interference between top-pair and single-top contributions.

A theoretically more rigorous approach consists of adopting the four-flavour (4F) scheme, where initial-state b-quarks result from gluons via explicit $\mathrm{g} \rightarrow \mathrm{b} \overline{\mathrm{b}}$ splittings. In this framework, the process $\mathrm{pp} \rightarrow \mathrm{W}^{+} \mathrm{W}^{-} \mathrm{b} \overline{\mathrm{b}}+X$ provides a unified description of $\mathrm{Wt}$ and $\mathrm{tt}$ production [2], and the presence of the $\mathrm{t} \overline{\mathrm{t}}-\mathrm{Wt}$ interference at LO stabilises the perturbative expansion. In the $4 \mathrm{~F}$ scheme, treating finite-top-width effects in the complex-mass scheme [3] ensures a consistent off-shell continuation of top-quark propagators and allows one to include double-, single-, and non-resonant contributions to $\mathrm{pp} \rightarrow \mathrm{W}^{+} \mathrm{W}^{-} \mathrm{b} \overline{\mathrm{b}}+X$ with all relevant interferences. Moreover, the ill-defined separation of top-pair and Wt production can be replaced by a gauge-invariant separation of $\mathrm{pp} \rightarrow \mathrm{W}^{+} \mathrm{W}^{-} \mathrm{b} \overline{\mathrm{b}}$ into its narrow-top-width limit, which corresponds to on-shell top-pair production and decay, and a finite-width remainder that includes off-shell $\overline{t t}$ effects as well as single-top and non-resonant contributions plus related interferences. 
The presence of four final-state particles and intermediate top-quark resonances render the simulation of $\mathrm{W}^{+} \mathrm{W}^{-} \mathrm{b} \overline{\mathrm{b}}$ production quite challenging beyond LO. First NLO calculations with massless b-quarks have been presented in [4-6]. For $\mathrm{W}^{+} \mathrm{W}^{-} \mathrm{b} \overline{\mathrm{b}}$ production with two hard $\mathrm{b}$-jets, apart from a few noticeable exceptions [5], most observables turn out to be completely dominated by the on-shell $\mathrm{tt}$ contribution. In phase-space regions with unresolved b-quarks, the importance of off-shell and single-top contributions is expected to increase quite substantially. However, due to the presence of collinear singularities, such regions are not accessible in the massless b-quark approximation of [4-6]. To fill this gap, in this paper we present a complete $\mathrm{NLO} \mathrm{W}^{+} \mathrm{W}^{-} \mathrm{b} \overline{\mathrm{b}}$ calculation including off-shell $\mathrm{W}$-boson decays and massive b-quarks in the $4 \mathrm{~F}$ scheme. A similar calculation has been presented very recently in [7]. These simulations provide NLO accurate $\mathrm{W}^{+} \mathrm{W}^{-} \mathrm{b} \overline{\mathrm{b}}$ predictions in the full phase space and allow one to investigate, for the first time, top-pair and single-top production in presence of jet vetoes or jet bins, such as in the case of the $\mathrm{H} \rightarrow \mathrm{W}^{+} \mathrm{W}^{-}$analysis. An important advantage of $\mathrm{NLO} \mathrm{W}^{+} \mathrm{W}^{-} \mathrm{b} \overline{\mathrm{b}}$ predictions in the $4 \mathrm{~F}$ scheme is that they provide a fully differential NLO description of both finalstate b-jets and a correspondingly accurate modelling of jet vetoes, while in the $5 \mathrm{~F}$ scheme a similar level of accuracy for spectator b-quarks in $\mathrm{Wt}$ production would require an NNLO calculation.

\section{Technical tools and ingredients of the calculation}

We will focus on NLO predictions for $\mathrm{pp} \rightarrow \nu_{\mathrm{e}} \mathrm{e}^{+} \mu^{-} \bar{v}_{\mu} \mathrm{b} \overline{\mathrm{b}}$, which comprises $t \bar{t}$ production and decay in the oppositeflavour di-lepton channel. For brevity we will denote this reaction as $\mathrm{W}^{+} \mathrm{W}^{-} \mathrm{b} \overline{\mathrm{b}}$ production, keeping in mind that all off-shell and interference effects related to the $\nu_{\mathrm{e}} \mathrm{e}^{+} \mu^{-} \bar{v}_{\mu}$ final state are consistently handled in the complex-mass scheme [3], where finite-width effects are systematically absorbed in the imaginary part of the renormalised pole mass. The complex-mass scheme is used also for the off-shell continuation of top-quark resonances [5]. Examples of tree diagrams involving two, one and no top-quark resonances are illustrated in Figs. 1 and 2. The second diagram in Fig. 1 is the $4 \mathrm{~F}$-scheme analog on of $t$-channel $\mathrm{gb} \rightarrow \mathrm{tW}^{-}$production in the $5 \mathrm{~F}$ scheme, and the initial-state $\mathrm{g} \rightarrow \mathrm{b} \bar{b}$ splitting is related to the b-quark parton distribution in 5F PDFs. At NLO we include the full set of tree, one-loop and real-emission diagrams that contribute to $v_{\mathrm{e}} \mathrm{e}^{+} \mu^{-} \bar{v}_{\mu} \mathrm{b} \overline{\mathrm{b}}$ production without applying any approximation. In particular non-resonant $Z / \gamma \rightarrow v_{\mathrm{e}} \mathrm{e}^{+} \mu^{-} \bar{v}_{\mu}$ sub-topologies like in the second diagram of Fig. 2 are included also in the virtual and real corrections. The bottom- and top-quark masses are renormalised in the on-shell scheme, and their contributions are retained everywhere.
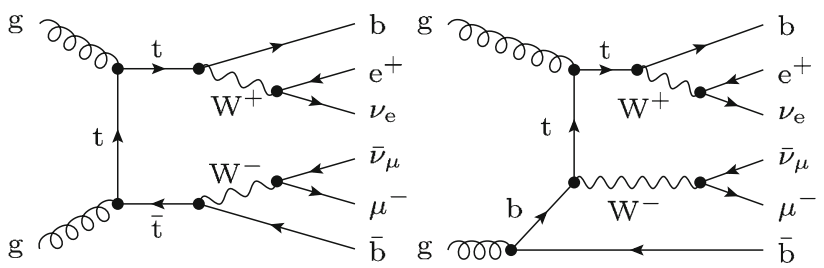

Fig. 1 Representative $\mathrm{t} \overline{\mathrm{t}}-$ like (left) and Wt-like (right) tree diagrams
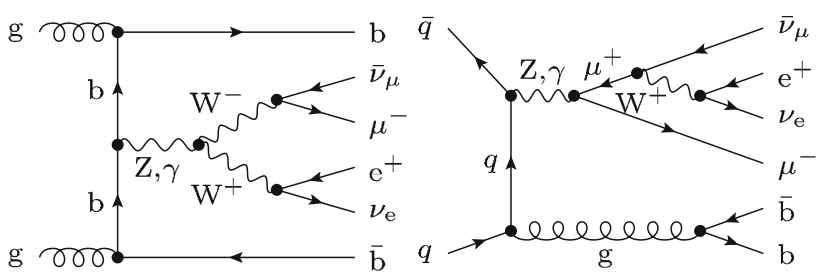

Fig. 2 Representative tree topologies without top resonances and with two (left) or only one (right) resonant W-boson

The entire calculation has been performed with highly flexible and automated NLO programs, and the high complexity resulting from the presence of multiple top- and $\mathrm{W}$ resonances, as well as from the wide spectrum of involved scales, render $\mathrm{pp} \rightarrow \mathrm{W}^{+} \mathrm{W}^{-} \mathrm{b} \overline{\mathrm{b}}$ an excellent technical benchmark to test the performance of the employed tools. To evaluate tree, virtual, and real-emission amplitudes, we employed OPENLOOPS [8], a new one-loop generator that will become public in the next future. The OPENLOOPS program is based on a novel numerical recursion, which is formulated in terms of loop-momentum polynomials called 'open loops' and allows for a fast evaluation of scattering amplitudes with many external particles. It uses the COLLIER library [9] for the numerically stable evaluation of tensor integrals $[10,11]$ and scalar integrals [12]. Together with [13,14], the present study is one of the very first applications of OPENLOOPS. Phasespace integration and infrared subtractions are performed with an in-house NLO Monte-Carlo framework [15], which is interfaced with OPENLOOPS and provides full automation along the entire chain of operations that are required for NLO calculations. This tool is applicable to any Standard-Model process at NLO QCD. Infrared singularities are handled with dipole subtraction [16,17], and since collinear $\mathrm{g} \rightarrow \mathrm{b} \overline{\mathrm{b}}$ splittings are regularised by the finite $b$-quark mass, corresponding subtraction terms are not included. The phase-space integrator is based on the adaptive multi-channel technique [18] and implements dedicated channels for the dipole subtraction terms, which improve the convergence, especially for multiresonance processes. Multiple scale variations in a single run are also supported. This tool has been validated in several NLO processes and, in combination with OPENLOOPS and COLLIER, it is also applicable to NNLO calculations [19]. The correctness of the results is supported by various checks: OPENLOOPS has been validated against an independent in- 
house generator for more than hundred partonic processes, including $\mathrm{W}^{+} \mathrm{W}^{-} \mathrm{b} \overline{\mathrm{b}}$ production with massless $\mathrm{b}$-quarks and various processes with massive heavy-quarks. For the process at hand we checked the cancellation of infrared and ultraviolet singularities. The correctness of phase-space integration and dipole subtraction was tested by means of a second calculation based on OPENLOOPS in combination with SHERPA [20,21] and AMEGIC++ [22].

\section{Input parameters, cuts and jet definition}

In the following, we present NLO results for $\mathrm{W}^{+} \mathrm{W}^{-} \mathrm{b} \overline{\mathrm{b}}$ production at the $8 \mathrm{TeV}$ LHC. For the heavy-quark and gaugeboson masses we use

$m_{\mathrm{t}}=173.2 \mathrm{GeV}, \quad m_{\mathrm{b}}=4.75 \mathrm{GeV}$,

$M_{\mathrm{Z}}=91.1876 \mathrm{GeV}, \quad M_{\mathrm{W}}=80.385 \mathrm{GeV}$.

The electroweak coupling is derived from the Fermi constant, $G_{\mu}=1.16637 \times 10^{-5} \mathrm{GeV}^{-2}$, in the $G_{\mu}$-scheme,

$\alpha=\frac{\sqrt{2}}{\pi} G_{\mu} M_{\mathrm{W}}^{2}\left(1-\frac{M_{\mathrm{W}}^{2}}{M_{\mathrm{Z}}^{2}}\right)$.

In the complex-mass scheme the electroweak mixing angle is evaluated as

$\cos ^{2} \theta_{\mathrm{w}}=\frac{M_{\mathrm{W}}^{2}-\mathrm{i} \Gamma_{\mathrm{W}} M_{\mathrm{W}}}{M_{\mathrm{Z}}^{2}-\mathrm{i} \Gamma_{\mathrm{Z}} M_{\mathrm{Z}}}$,

and for the widths we use the NLO QCD values

$\Gamma_{\mathrm{W}}=2.09530 \mathrm{GeV}, \quad \Gamma_{\mathrm{Z}}=2.50479 \mathrm{GeV}$

everywhere, i.e. for LO as well as for NLO matrix elements. The Higgs-boson mass and width are set to $M_{\mathrm{H}}=126 \mathrm{GeV}$ and $\Gamma_{\mathrm{H}}=4.21 \mathrm{MeV}$. To guarantee consistent top-decay branching fractions, matrix elements and top-width input parameters must be taken at the same perturbative order. For the LO and NLO top-quark widths we use the values

$\Gamma_{\mathrm{t}}^{\mathrm{LO}}=1.47451 \mathrm{GeV}, \quad \Gamma_{\mathrm{t}}^{\mathrm{NLO}}=1.34264 \mathrm{GeV}$,

which are computed with massive b-quarks and off-shell Wbosons [23]. Consistently with the use of massive b-quarks we employ 4F parton distributions. Specifically, at NLO the LHApdf implementation of the 4F NNPDF2.3 parton distributions [24] and the corresponding running strong coupling are used. More precisely, we use a reference set ${ }^{1}$ that is obtained from a variable-flavour set with $\alpha_{\mathrm{s}}^{(5)}\left(M_{\mathrm{Z}}\right)=0.118$ via inverse $5 \mathrm{~F}$ evolution down to $\mu_{\mathrm{F}}=m_{\mathrm{b}}$ and subsequent upward evolution with four active flavours. Since the NNPDF2.3 release does not include LO parton distributions, for LO predictions we adopt the NNPDF21_lo_nf4_100 4F

${ }^{1}$ NNPDF23_nlo_FFN_NF4_as_0118. set, which corresponds to a reference strong-coupling value $\alpha_{\mathrm{S}}^{(5)}\left(M_{\mathrm{Z}}\right)=0.119$. While the $4 \mathrm{~F}$ running of $\alpha_{\mathrm{S}}$ misses heavyquark-loop effects, corresponding $\mathcal{O}\left(\alpha_{\mathrm{s}}\right)$ contributions are consistently included in the virtual corrections via zeromomentum subtraction of the top- and bottom-quark loops in the renormalisation of $\alpha_{\mathrm{s}}$.

To investigate NLO corrections to top-pair and Wt production we select events with two oppositely charged leptons, $\ell=\mathrm{e}^{+}, \mu^{-}$, with

$p_{\mathrm{T}, \ell}>20 \mathrm{GeV}, \quad\left|\eta_{\ell}\right|<2.5, \quad p_{\mathrm{T}, \mathrm{miss}}>20 \mathrm{GeV}$,

where $p_{\mathrm{T}, \text { miss }}$ is obtained from the vector sum of the neutrinos' transverse momenta. Final-state QCD partons, including b-quarks, are recombined into IR-safe jets using the anti- $k_{\mathrm{T}}$ algorithm [25] with jet-resolution parameter $R=0.4$. Events are categorised according to the total number, $N_{j}$, of jets with $p_{\mathrm{T}}>30 \mathrm{GeV}$ and $|\eta|<2.5$ and the number of b-jets, $N_{\mathrm{b}}$, within the same acceptance region. We classify as b-jet any jet involving at least a b-quark, which includes also the case of collimated $b \bar{b}$ pairs resulting from the splitting of energetic gluons. In fixed-order calculations the implementation of this b-jet definition is possible only in presence of mas-

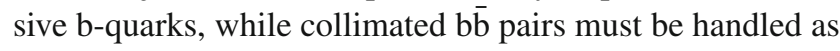
'gluon jets' in the massless case.

\section{Scale choice for top-pair and single-top production}

In order to isolate off-shell and single-top effects associated with the finite top-quark width (FtW) we decompose the differential $\mathrm{W}^{+} \mathrm{W}^{-} \mathrm{b} \overline{\mathrm{b}}$ cross section as

$\mathrm{d} \sigma_{\mathrm{W}^{+} \mathrm{W}-\mathrm{b} \overline{\mathrm{b}}}=\mathrm{d} \sigma_{\mathrm{t} \overline{\mathrm{t}}}+\mathrm{d} \sigma_{\mathrm{W}^{+} \mathrm{W}-\mathrm{b} \overline{\mathrm{b}}}^{\mathrm{FtW}}$,

where the t⿱t term represents on-shell top-pair production and

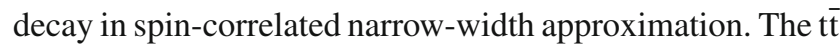
contribution is obtained from the numerical extrapolation of the full $\mathrm{W}^{+} \mathrm{W}^{-} \mathrm{b} \overline{\mathrm{b}}$ cross section in the narrow-width limit [5],

$\mathrm{d} \sigma_{\mathrm{tt}}=\lim _{\Gamma_{\mathrm{t}} \rightarrow 0} \mathrm{~d} \tilde{\sigma}_{\mathrm{W}^{+} \mathrm{W}^{-} \mathrm{b} \overline{\mathrm{b}}}\left(\Gamma_{\mathrm{t}}\right)$,

with

$\mathrm{d} \tilde{\sigma}_{\mathrm{W}^{+} \mathrm{W}^{-} \mathrm{b} \overline{\mathrm{b}}}\left(\Gamma_{\mathrm{t}}\right)=\left(\frac{\Gamma_{\mathrm{t}}}{\Gamma_{\mathrm{t}}^{\text {phys }}}\right)^{2} \mathrm{~d} \sigma_{\mathrm{W}^{+} \mathrm{W}^{-} \mathrm{b} \overline{\mathrm{b}}}\left(\Gamma_{\mathrm{t}}\right)$,

where the factor $\left(\Gamma_{t} / \Gamma_{t}^{\text {phys }}\right)^{2}$ compensates the $1 / \Gamma_{t}^{2}$ scaling of the cross section in such a way that top-decay branching fractions remain constant when $\Gamma_{\mathrm{t}} \rightarrow 0$. By construction the $\mathrm{d} \sigma_{\mathrm{W}^{+} \mathrm{W}^{-} \mathrm{bb}}^{\mathrm{FtW}}$ remainder in (7) contains all finite-top-width effects, including off-shell tt production as well as single-top and non-resonant contributions.

As compared to $\mathrm{W}^{+} \mathrm{W}^{-} \mathrm{b} \overline{\mathrm{b}}$ production with two hard $\mathrm{b}$ jets, the fully inclusive case involves a much wider spectrum of scales, ranging from $m_{\mathrm{b}}$ to $m_{\mathrm{t} \mathrm{t}}$. This renders theoretical 
calculations significantly more involved. In particular, given that the $\mathrm{t} \overline{\mathrm{t}}$ and $\mathrm{Wt}$ contributions to $\mathrm{W}^{+} \mathrm{W}^{-} \mathrm{b} \overline{\mathrm{b}}$ production are characterised by very different scales, it is a priori not clear if a conventional QCD scale choice can ensure a perturbatively stable description of both contributions. For tt production, a scale of the order of the geometric average of the top-quark transverse energies,

$\mu_{\mathrm{tt}}^{2}=E_{\mathrm{T}, \mathrm{t}} E_{\mathrm{T}, \overline{\mathrm{t}}} \quad$ with $\quad E_{\mathrm{T}, i}^{2}=m_{i}^{2}+p_{\mathrm{T}, i}^{2}$,

is known to ensure a good perturbative convergence [5]. In the case of the single-top $\mathrm{W}^{-} \mathrm{t}$ contribution one has to deal with two sub-processes: a collinear $g \rightarrow$ b $\bar{b}$ initial-state splitting followed by $\mathrm{gb} \rightarrow \mathrm{W}^{-}$t hard scattering. ${ }^{2}$ The respective characteristic scales are the bottom- and the top-quark transverse energies, $E_{\mathrm{T}, \mathrm{b}} \ll E_{\mathrm{T}, \mathrm{t}}$, and a QCD scale of type

$\mu_{\mathrm{tW}^{-}}^{2}=E_{\mathrm{T}, \mathrm{t}} E_{\mathrm{T}, \overline{\mathrm{b}}}$

should represent an appropriate choice, since

$\alpha_{\mathrm{s}}^{2}\left(\mu_{\mathrm{tW}^{-}}^{2}\right) \simeq \alpha_{\mathrm{s}}\left(E_{\mathrm{T}, \mathrm{t}}^{2}\right) \alpha_{\mathrm{S}}\left(E_{\mathrm{T}, \overline{\mathrm{b}}}^{2}\right)$

guarantees that the $\alpha_{\mathrm{s}}$ factor associated with the collinear $\mathrm{g} \rightarrow \mathrm{b} \overline{\mathrm{b}}$ splitting is effectively evaluated at the scale $E_{\mathrm{T}, \mathrm{b}}$, similarly as in the resummation of initial-state b-quark emissions in the evolution of 5F PDFs. Vice versa, using a global QCD scale of the order $m_{\mathrm{t}}$ might underestimate the single-top component of $\mathrm{pp} \rightarrow \mathrm{W}^{+} \mathrm{W}^{-} \mathrm{b} \overline{\mathrm{b}}$ by up to a factor $\alpha_{\mathrm{s}}\left(m_{\mathrm{b}}\right) / \alpha_{\mathrm{s}}\left(m_{\mathrm{t}}\right) \sim 2$ at LO. This would be compensated by $\ln \left(m_{\mathrm{b}}\right)$-enhanced higher-order corrections, resulting in a poor perturbative convergence. For an accurate description of the single-top contribution, the above considerations motivate a dynamic QCD scale that interpolates between (10) and (11) in t $\bar{t}-$ and Wt-dominated regions, respectively. Such a scale can be defined as

$\mu_{\mathrm{WWbb}}^{2}=\mu_{\mathrm{W}+\mathrm{b}} \mu_{\mathrm{W}-\overline{\mathrm{b}}}$,

with

$\mu_{\mathrm{Wb}}=P_{\mathrm{b}}\left(p_{\mathrm{W}}, p_{\mathrm{b}}\right) E_{\mathrm{T}, \mathrm{b}}+P_{\mathrm{t}}\left(p_{\mathrm{W}}, p_{\mathrm{b}}\right) E_{\mathrm{T}, \mathrm{t}}$,

where $\mathrm{Wb}$ represents either $\mathrm{W}^{+} \mathrm{b}$ or $\mathrm{W}^{-} \overline{\mathrm{b}}$, and the functions $P_{\mathrm{b}}$ and $P_{\mathrm{t}}=1-P_{\mathrm{b}}$ describe the probability that the b-quark of a given $\mathrm{Wb}$ pair arises from an initial-state $\mathrm{g} \rightarrow \mathrm{b} \overline{\mathrm{b}}$ splitting or from at $\rightarrow \mathrm{Wb}$ decay, respectively. Their approximate functional form can be obtained from the leading matrix-element singularities associated with the $g \rightarrow b \bar{b}$ and $\mathrm{t} \rightarrow \mathrm{Wb}$ sub-processes, ${ }^{3}$

$\chi_{\mathrm{b}}=\frac{m_{\mathrm{t}}^{2}}{E_{\mathrm{T}, \mathrm{b}}^{2}}, \quad \chi_{\mathrm{t}}=\frac{m_{\mathrm{t}}^{4}}{\left[\left(p_{\mathrm{W}}+p_{\mathrm{b}}\right)^{2}-m_{\mathrm{t}}^{2}\right]^{2}+\Gamma_{\mathrm{t}}^{2} m_{\mathrm{t}}^{2}}$,

\footnotetext{
2 The charge-conjugate channels are implicitly understood.

3 The $\chi_{\mathrm{b}}$ and $\chi_{\mathrm{t}}$ distributions are defined as dimensionless functions by introducing $m_{\mathrm{t}}$-terms in the numerator. This convention is, however, irrelevant, since the probabilities resulting from (16) and (17) are independent of the normalisation of $\chi_{\mathrm{b}}$ and $\chi_{\mathrm{t}}$.
}

by requiring that $P_{\mathrm{b}} / P_{\mathrm{t}} \propto \chi_{\mathrm{b}} / \chi_{\mathrm{t}}$. This yields

$P_{\mathrm{b}}=1-P_{\mathrm{t}}=\frac{\chi_{\mathrm{b}}}{\chi_{\mathrm{b}}+R \chi_{\mathrm{t}}}$.

The constant $R$ can be derived from the condition

$\int \mathrm{d} \sigma_{\mathrm{W}^{+} \mathrm{W}^{-} \mathrm{b} \overline{\mathrm{b}}}^{\mathrm{FtW}}=\int \mathrm{d} \Phi\left[1-P_{\mathrm{t}}(\Phi) P_{\mathrm{t}}(\Phi)\right] \frac{\mathrm{d} \sigma_{\mathrm{W}^{+} \mathrm{W}^{-} \mathrm{b} \overline{\mathrm{b}}}}{\mathrm{d} \Phi}$,

i.e. by requiring that finite-top-width corrections to the inclusive $\mathrm{W}^{+} \mathrm{W}^{-} \mathrm{b} \overline{\mathrm{b}}$ cross section correspond to the contribution from non- $t \bar{t}$ events according to the probability distributions $P_{\mathrm{b}}$ and $P_{\mathrm{t}}{ }^{4}$ The tuning of $R$ is performed in LO approximation on the fully inclusive level and yields $R=7.96$. At NLO, the kinematic quantities that enter $\mu_{\mathrm{Wwbb}}$ are defined in terms of $b$ - and $\bar{b}$-jet momenta that are constructed with a modified jet algorithm where b $\bar{b}$ pairs are not clustered and light partons with $|\eta|>4.5$ are excluded from the recombination procedure. The latter prescription guarantees the collinear safety of the reconstructed top mass, $\left(p_{\mathrm{W}}+p_{\mathrm{b}}\right)^{2}$, with respect to collinear light-parton emission from the initial state. In the reconstruction of the top and anti-top masses $\left(p_{\mathrm{W}}+p_{\mathrm{b}}\right)^{2}$ that enter (15), remaining hard jets are clustered with the $\mathrm{t}-$ or $\overline{\mathrm{t}}-\mathrm{system}$ if the resulting invariant mass turns out to be closer to $m_{\mathrm{t}}$. Top-jet clusterings are applied only if they yield $P_{\mathrm{t}}>0.5$. If that holds for $\mathrm{t}-$ and $\overline{\mathrm{t}}-$ system, the clustering to maximise the tit probability, $P_{\mathrm{t}} P_{\mathrm{t}}$, is chosen.

\section{Predictions for the $\mathrm{LHC}$ at $8 \mathrm{TeV}$}

In the following we present predictions for $\mathrm{pp} \rightarrow \mathrm{W}^{+} \mathrm{W}^{-} \mathrm{b} \overline{\mathrm{b}}$ at $8 \mathrm{TeV}$ in presence of the leptonic cuts (6). If not stated otherwise, the renormalisation and factorisation scales are set to

$\mu_{\mathrm{R}, \mathrm{F}}=\xi_{\mathrm{R}, \mathrm{F}} \mu_{0}$ with $\mu_{0}=\mu_{\mathrm{WWbb}}$,

where $\xi_{\mathrm{R}}=\xi_{\mathrm{F}}=1$ corresponds to the default scale choice. Theoretical uncertainties are assessed by applying the scale variations $\left(\xi_{\mathrm{R}}, \xi_{\mathrm{F}}\right)=(2,2),(2,1),(1,2),(1,0.5),(0.5,1)$, $(0.5,0.5)$.

Figure 3 illustrates the extrapolation of the $\mathrm{W}^{+} \mathrm{W}^{-} \mathrm{b} \overline{\mathrm{b}}$ cross section in the narrow-top-width limit (7)-(8). The results are well consistent-at the few-permil level-with the expected linear convergence of the NLO cross section in the $\Gamma_{\mathrm{t}} \rightarrow 0$ limit. This provides a non-trivial check of the consistency of the calculation, since the narrow-width limit involves delicate cancellations of logarithmic singularities that arise from virtual and real soft-gluon corrections to the resonant top-quark propagators. Finite-width effects turn

\footnotetext{
${ }^{4}$ Here we assume that finite-top-width effects are dominated by non-t $\bar{t}$ contributions. Note also that the finite-top-width term on the left-hand side of (17) must be extracted through $\Gamma_{\mathrm{t}} \rightarrow 0$ extrapolation by keeping $\Gamma_{\mathrm{t}}$ and $R$ fixed in (15)-(16).
} 


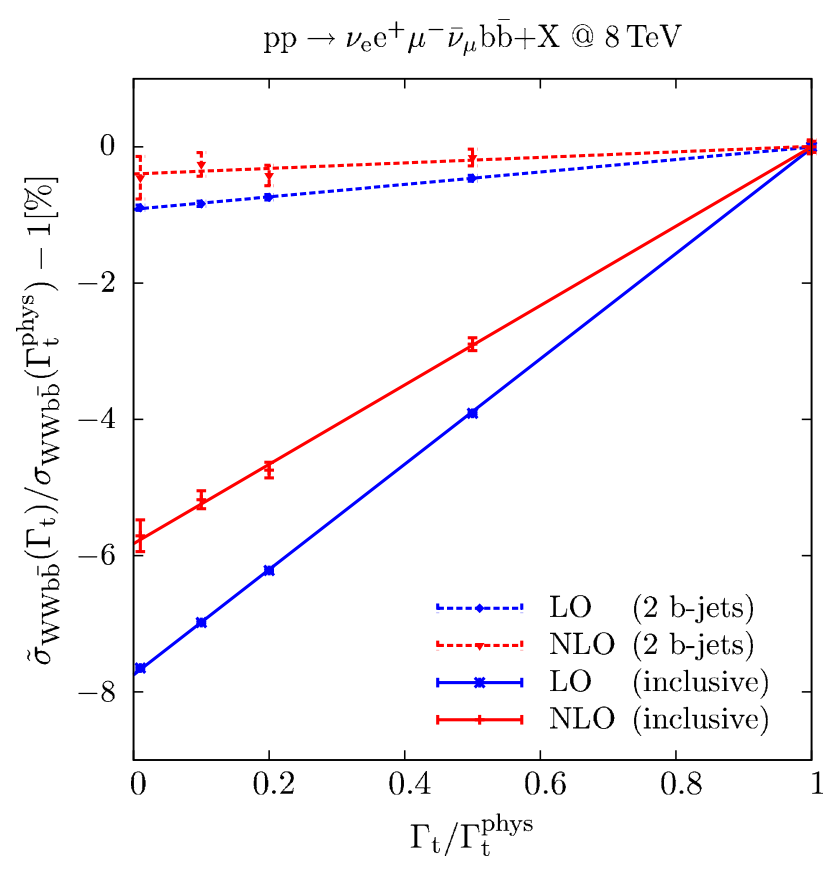

Fig. 3 Numerical extrapolation of the $\mathrm{LO}$ and $\mathrm{NLO} \mathrm{W}^{+} \mathrm{W}^{-} \mathrm{b} \overline{\mathrm{b}}$ cross section with leptonic cuts in the narrow-top-width limit, $\Gamma_{t} \rightarrow 0$. Results are shown as relative deviations (in percent) with respect to the $\mathrm{W}^{+} \mathrm{W}^{-} \mathrm{b} \overline{\mathrm{b}}$ cross section with $\Gamma_{\mathrm{t}}=\Gamma_{\mathrm{t}}^{\text {phys }}$. Results with inclusive jet emission are compared to a $\overline{\mathrm{t}}$-signal analysis with two b-jets

out to be at the sub-percent level if one requires the presence of two b-jets, like in a typical $\mathrm{t} \overline{\mathrm{t}}$-signal analysis. For the total cross section they are instead clearly more important. Their net effect, which results from the interplay of negative off-shell corrections and positive single-top contributions, amounts to about $+6 \%(8 \%)$ at $\mathrm{NLO}(\mathrm{LO})$.

Predictions for the integrated cross section and in exclusive jet bins are listed in Table 1. To assess the influence of the scale choice, results based on $\mu_{0}=\mu_{\mathrm{Wwbb}}$ are compared to the case of the conventional scale $\mu_{0}=m_{\mathrm{t}}$. For the total cross section we find positive corrections of about $40 \% .^{5}$

Scale uncertainties decrease from about $30 \%$ at $\mathrm{LO}$ to $10 \%$ at NLO, and the differences between the two scale choices are consistent within scale variations. The last three columns of Table 1 display jet cross sections in bins with $N_{j}=0,1$ and $N_{j} \geq 2$ jets, where $N_{j}$ refers to the total number of b-jets and light jets. The different bins receive quite

\footnotetext{
5 We note that these results are not directly comparable to those of [5], which reports a significantly smaller $K$-factor. In particular, while we apply the same cuts on leptons, missing energy and jets, here we do not restrict ourselves to the case of two b-jets, we adopt a smaller jetresolution parameter and a different QCD scale choice. Moreover we employ a 4F PDF set, which implies an enhancement of the gluon density due to the absence of $\mathrm{g} \rightarrow \mathrm{b} \bar{b}$ splittings in the PDF evolution. The LO PDF sets used in [5] and in the present study feature also significantly different values of $\alpha_{\mathrm{s}}$, which influences LO results and $K$ factors. Finally, in addition to uniform scale variations considered in [5], here also independent $\mu_{\mathrm{R}}$ and $\mu_{\mathrm{F}}$ variations are taken into account.
}

different corrections, and the relative weight of the individual bins in percent changes from 3:30:67 at LO to 2:21:76 at NLO. This indicates that a significant fraction of the 0 - and 1 -jet bin cross sections migrates to the inclusive 2 -jet bin. We attribute this feature to the rather high probability of light-jet emissions with $p_{\mathrm{T}} \gtrsim 30 \mathrm{GeV}$. While NLO scale uncertainties turn out to be fairly small in all jet bins, matching to the parton shower is certainly important for a more reliable description of such radiative processes. Comparing the two scale choices, also in jet bins we do not observe any dramatic difference: absolute LO and NLO results are well consistent within scale variations, and also $\mathrm{K}$-factors and scale variations themselves turn out to be quite similar.

Finite-top-width $(\mathrm{FtW})$ contributions are shown in the lower part of Table 1 . For what concerns the total $\mathrm{W}^{+} \mathrm{W}^{-} \mathrm{b} \overline{\mathrm{b}}$ cross section their impact is around $6 \%$, and the scale $\mu_{\mathrm{Wwbb}}$ guarantees a good perturbative convergence: FtW contributions receive only minor NLO corrections, and the residual scale dependence is about $10 \%$, while setting $\mu_{0}=m_{\mathrm{t}}$ yields larger corrections and scale uncertainties. As compared to complete $\mathrm{W}^{+} \mathrm{W}^{-} \mathrm{b} \overline{\mathrm{b}}$ predictions, FtW contributions are distributed in a completely different way among jet bins. The relative weight in percent of the 0 -, 1- and 2-jet bins is 14:78:8 at LO and 12:57:31 at NLO. These results suggest that FtW effects are dominated by a single-top Wt component, which is concentrated in the 1 -jet bin at $\mathrm{LO}$ and tends to migrate to the 2-jet bin due to light-jet emissions at NLO. The fact that the FtW part of the 2-jet bin features a 40-50\% NLO uncertainty is irrelevant, since this contribution represents less than $3 \%$ of the complete cross section in the 2-jet bin. In the 0 - and 1-jet bins, whose FtW components amount to 32 and $16 \%$, respectively, NLO scale uncertainties are as small as $10 \%$ or so.

In Table 2 we report analogous results for the $\mathrm{W}^{+} \mathrm{W}^{-} \mathrm{b} \overline{\mathrm{b}}$ cross section and its FtW contribution in b-jet bins. As compared to the case of generic jets, we observe that $\mathrm{W}^{+} \mathrm{W}^{-} \mathrm{b} \overline{\mathrm{b}}$ $\mathrm{K}$-factors feature a less pronounced dependence on the $\mathrm{b}$ jet multiplicity if the $\mu_{\mathrm{WWbb}}$ scale is used. This is due to the fact that NLO emissions consist of light jets and are thus less likely to induce bin migrations in the case of bjet bins. Scale uncertainties at NLO are at the 20, 15 and $10 \%$ level in the bins with 0,1 , and $\geq 2$ b-jets, respectively. Finite-top-width contributions turn out to be even more stable than full $\mathrm{W}^{+} \mathrm{W}^{-} \mathrm{b} \overline{\mathrm{b}}$ results with the scale $\mu_{\mathrm{WWbb}}$, while the scale $m_{\mathrm{t}}$ tends to give larger uncertainties. Using the $\mu_{\mathrm{WWbb}}$ scale, FtW effects in the 0-, 1-, and 2-b-jet bins turn out to be 31,14 and 0.4 percent of the respective $\mathrm{W}^{+} \mathrm{W}^{-} \mathrm{b} \overline{\mathrm{b}}$ cross sections at NLO. Employing $\mu_{0}=m_{\mathrm{t}}$ these percentages become 25,13 and 0.5 , respectively. In general, jet- and b-jet-bin results indicate that the conventional scale $\mu_{0}=m_{\mathrm{t}}$ yields a similarly good perturbative convergence as $\mu_{0}=\mu_{\text {wwbb }}$. However, it is a priori not clear if this holds also for more exclusive observables. For 
Table 1 LO and NLO predictions for $\mathrm{pp} \rightarrow \mathrm{W}^{+} \mathrm{W}^{-} \mathrm{b} \overline{\mathrm{b}}$ and corrections, scale choices: total cross section with leptonic cuts and partial contributions with 0,1 and $\geq 2$ jets. Full $\mathrm{W}^{+} \mathrm{W}^{-} \mathrm{b} \overline{\mathrm{b}}$ predictions $(\sigma)$ are compared to finite-top-width contributions $\left(\sigma^{\mathrm{FtW}}\right)$ at $8 \mathrm{TeV}$ with scale variations

$K=\sigma_{\mathrm{NLO}} / \sigma_{\mathrm{LO}}$, for different

\begin{tabular}{llllll}
\hline & $\mu_{0}$ & $\sigma[\mathrm{fb}]$ & $\sigma_{0}[\mathrm{fb}]$ & $\sigma_{1}[\mathrm{fb}]$ & $\sigma_{2^{+}}[\mathrm{fb}]$ \\
\hline $\mathrm{LO}$ & $\mu_{\mathrm{WWbb}}$ & $1232_{-24 \%}^{+34 \%}$ & $37_{-25 \%}^{+38 \%}$ & $367_{-24 \%}^{+36 \%}$ & $828_{-23 \%}^{+33 \%}$ \\
$\mathrm{NLO}$ & $\mu_{\mathrm{WWbb}}$ & $1777_{-12 \%}^{+10 \%}$ & $41_{-8 \%}^{+3 \%}$ & $377_{-6 \%}^{+1 \%}$ & $1359_{-14 \%}^{+14 \%}$ \\
$K$ & $\mu_{\mathrm{WWbb}}$ & 1.44 & 1.09 & 1.03 & 1.64 \\
$\mathrm{LO}$ & $m_{\mathrm{t}}$ & $1317_{-24 \%}^{+35 \%}$ & $35_{-25 \%}^{+37 \%}$ & $373_{-24 \%}^{+36 \%}$ & $909_{-24 \%}^{+35 \%}$ \\
$\mathrm{NLO}$ & $m_{\mathrm{t}}$ & $1817_{-11 \%}^{+8 \%}$ & $40_{-8 \%}^{+4 \%}$ & $372_{-8 \%}^{+1 \%}$ & $1405_{-13 \%}^{+13 \%}$ \\
$K$ & $m_{\mathrm{t}}$ & 1.38 & 1.14 & 1.00 & 1.55 \\
\hline & $\mu_{0}$ & $\sigma^{\mathrm{FtW}}[\mathrm{fb}]$ & $\sigma_{0}^{\mathrm{FtW}}[\mathrm{fb}]$ & $\sigma_{1}^{\mathrm{FtW}}[\mathrm{fb}]$ & $\sigma_{2+}^{\mathrm{FtW}}[\mathrm{fb}]$ \\
\hline $\mathrm{LO}$ & $\mu_{\mathrm{WWbb}}$ & $91_{-27 \%}^{+41 \%}$ & $13_{-27 \%}^{+42 \%}$ & $71_{-27 \%}^{+40 \%}$ & $7_{-29 \%}^{+45 \%}$ \\
$\mathrm{NLO}$ & $\mu_{\mathrm{WWbb}}$ & $107_{-11 \%}^{+66 \%}$ & $13_{-7 \%}^{+1 \%}$ & $61_{-16 \%}^{+2 \%}$ & $33_{-31 \%}^{+51 \%}$ \\
$K$ & $\mu_{\mathrm{WWbb}}$ & 1.18 & 0.99 & 0.86 & 4.70 \\
$\mathrm{LO}$ & $m_{\mathrm{t}}$ & $63_{-25 \%}^{+36 \%}$ & $8_{-25 \%}^{+36 \%}$ & $49_{-24 \%}^{+36 \%}$ & $6_{-29 \%}^{+46 \%}$ \\
$\mathrm{NLO}$ & $m_{\mathrm{t}}$ & $100_{-16 \%}^{+17 \%}$ & $13_{-14 \%}^{+14 \%}$ & $65_{-12 \%}^{+9} \%$ & $23_{-28 \%}^{+42 \%}$ \\
$K$ & $m_{\mathrm{t}}$ & 1.58 & 1.47 & 1.32 & 3.89 \\
\hline
\end{tabular}

Table 2 Full $\mathrm{W}^{+} \mathrm{W}^{-} \mathrm{b} \overline{\mathrm{b}}$ predictions and finite-top-width contributions for bins with 0,1 and $\geq 2$ b-jets. Same conventions as in Table 1

\begin{tabular}{|c|c|c|c|c|c|}
\hline & $\mu_{0}$ & $\sigma[\mathrm{fb}]$ & $\sigma_{0}[\mathrm{fb}]$ & $\sigma_{1}[\mathrm{fb}]$ & $\sigma_{2^{+}}[\mathrm{fb}]$ \\
\hline LO & $\mu_{\mathrm{WWbb}}$ & $1232_{-24 \%}^{+34 \%}$ & $37_{-25 \%}^{+38 \%}$ & $367_{-24 \%}^{+36 \%}$ & $828_{-23 \%}^{+33 \%}$ \\
\hline NLO & $\mu_{\mathrm{WWbb}}$ & $1777_{-12 \%}^{+10 \%}$ & $65_{-17 \%}^{+20 \%}$ & $571_{-14 \%}^{+14 \%}$ & $1140_{-10 \%}^{+7 \%}$ \\
\hline$K$ & $\mu_{\mathrm{WWbb}}$ & 1.44 & 1.73 & 1.56 & 1.38 \\
\hline LO & $m_{\mathrm{t}}$ & $1317_{-24 \%}^{+35 \%}$ & $35_{-25 \%}^{+37 \%}$ & $373_{-24 \%}^{+36 \%}$ & $909_{-24 \%}^{+35 \%}$ \\
\hline NLO & $m_{\mathrm{t}}$ & $1817_{-11 \%}^{+8 \%}$ & $63_{-17 \%}^{+20 \%}$ & $584_{-14 \%}^{+14 \%}$ & $1170_{-9}^{+5 \%}$ \\
\hline \multirow[t]{2}{*}{$K$} & $m_{\mathrm{t}}$ & 1.38 & 1.80 & 1.56 & 1.29 \\
\hline & $\mu_{0}$ & $\sigma^{\mathrm{FtW}}[\mathrm{fb}]$ & $\sigma_{0}^{\mathrm{FtW}}[\mathrm{fb}]$ & $\sigma_{1}^{\mathrm{FtW}}[\mathrm{fb}]$ & $\sigma_{2^{+}}^{\mathrm{FtW}}[\mathrm{fb}]$ \\
\hline LO & $\mu_{\mathrm{WWbb}}$ & $91_{-27 \%}^{+41 \%}$ & $13_{-27 \%}^{+42 \%}$ & $71_{-27 \%}^{+40 \%}$ & $7_{-29 \%}^{+45 \%}$ \\
\hline NLO & $\mu_{\mathrm{WWbb}}$ & $107_{-11 \%}^{+6 \%}$ & $20_{-17 \%}^{+18 \%}$ & $82_{-10 \%}^{+4 \%}$ & $5_{-10 \%}^{+2 \%}$ \\
\hline$K$ & $\mu_{\mathrm{WWbb}}$ & 1.18 & 1.49 & 1.16 & 0.77 \\
\hline LO & $m_{\mathrm{t}}$ & $63_{-25 \%}^{+36 \%}$ & $8_{-25 \%}^{+36 \%}$ & $49_{-24 \%}^{+36 \%}$ & $6_{-29 \%}^{+46 \%}$ \\
\hline NLO & $m_{\mathrm{t}}$ & $100_{-16 \%}^{+17 \%}$ & $16_{-18 \%}^{+22 \%}$ & $77_{-15 \%}^{+16 \%}$ & $6_{-16 \%}^{+12 \%}$ \\
\hline$K$ & $m_{\mathrm{t}}$ & 1.58 & 1.89 & 1.58 & 1.10 \\
\hline
\end{tabular}

what concerns theoretical uncertainties in jet and b-jet bins, we checked that NLO scale variations remain similarly small as in Tables 1, 2 if the jet-rapidity acceptance is increased up to $|\eta|<4.5$.

To illustrate jet-veto and jet-binning effects in more detail, in Fig. 4 we plot the integrated $\mathrm{W}^{+} \mathrm{W}^{-} \mathrm{b} \overline{\mathrm{b}}$ cross section in exclusive bins with $N_{j}=0$ and $N_{j}=1$ jets versus the $p_{\mathrm{T}}$-threshold that defines jets. The 0 -jet bin corresponds to the integrated cross section in presence of a jet veto, $p_{\mathrm{T}, \text { jet }}<p_{\mathrm{T} \text {, jet }}^{\text {thr }}$. At large $p_{\mathrm{T} \text {, jet }}^{\text {thr }}$ the $K$-factor and the FtW contributions converge quite smoothly towards their inclusive limit. In contrast, the region of small transverse momentum features a very pronounced dependence on $p_{\mathrm{T}, \mathrm{jet}}^{\text {thr }}$ : FtW corrections grow from $6 \%$ up to more than $40 \%$, and the $K$-factor decreases very fast due to the presence of a soft singularity at $p_{\mathrm{T}, \text { jet }}^{\text {thr }} \rightarrow 0$. For a jet veto with $p_{\mathrm{T}, \text { jet }}^{\text {thr }}=30 \mathrm{GeV}$ we observe a $98 \%$ suppression of the $\mathrm{W}^{+} \mathrm{W}^{-} \mathrm{b} \overline{\mathrm{b}}$ cross section. Yet the moderate size of the $K$-factor and NLO scale variations indicates that the perturbative expansion is still rather stable in this regime. In the 1-jet bin, the limit of small $p_{\mathrm{T}, \mathrm{jet}}^{\mathrm{thr}}$ is driven by the effect of the veto on the second jet, and NLO and FtW corrections behave rather similarly as for the 0 -jet bin in this region. In the opposite regime, $p_{\mathrm{T}, \text { jet }}^{\text {thr }}$ mainly acts as a lower $p_{\mathrm{T}}$ bound for the first jet, and $\overline{\mathrm{t}}$ production with LO on-shell kinematics turns out to be kinemat- 


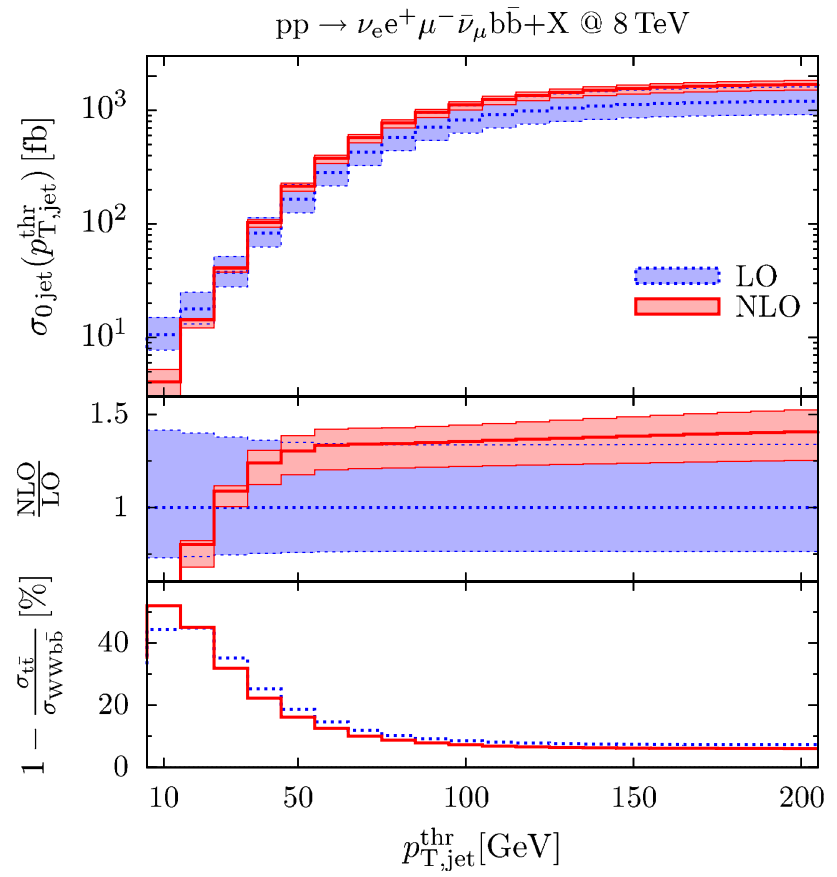

Fig. $4 \mathrm{LO}$ and $\mathrm{NLO} \mathrm{W}^{+} \mathrm{W}^{-} \mathrm{b} \overline{\mathrm{b}}$ cross sections in the exclusive bins with $N_{j}=0$ (left) and $N_{j}=1$ (right) jets as functions of the jet$p_{\mathrm{T}}$ threshold, $p_{\mathrm{T}, \mathrm{jet}}^{\mathrm{thr}}$. The middle of each bin corresponds to the actual value of $p_{\mathrm{T}, \mathrm{jet}}^{\mathrm{thr}}$. The central and lower frames show the $K$-factor and

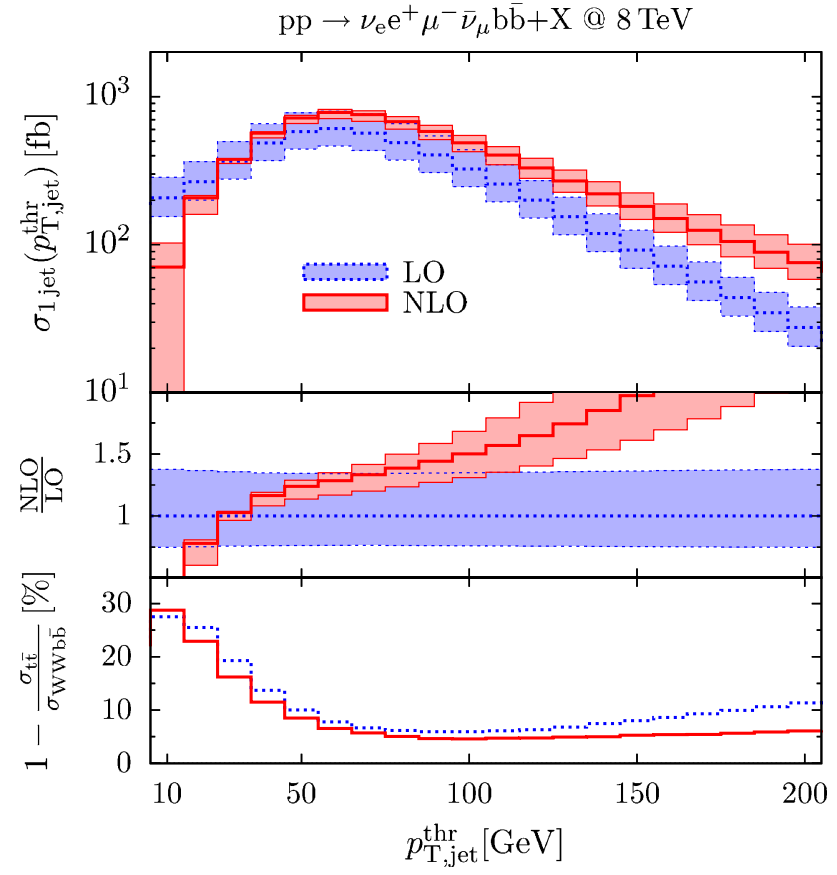

the relative impact in percent of finite-top-width contributions. Where depicted, bands correspond to independent scale variations of $\mu_{\mathrm{R}, \mathrm{F}}$ by a factor of two around the central scale $\mu_{\mathrm{wwbb}}$, not taking into account antipodal variations
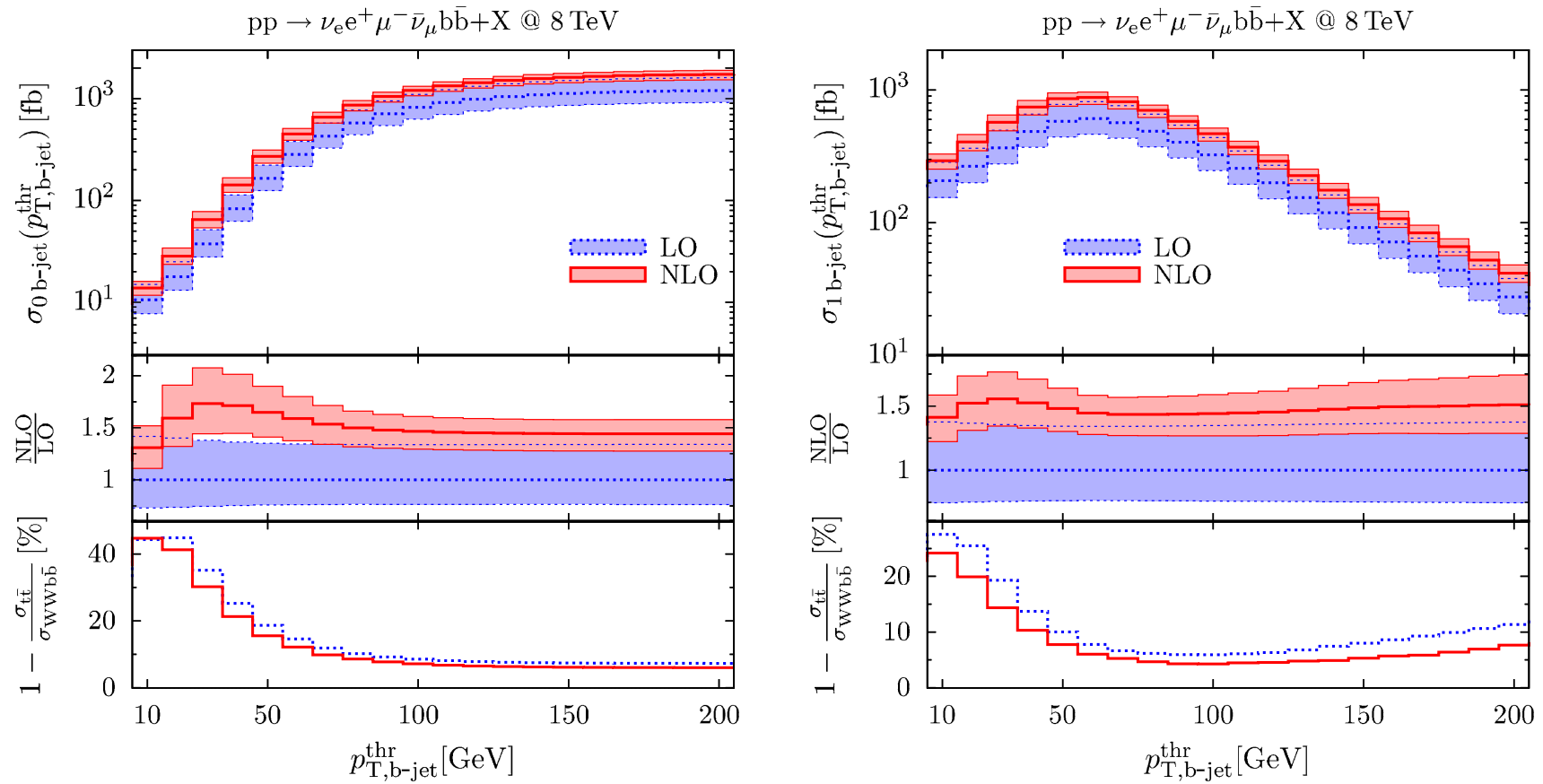

Fig. $5 \mathrm{LO}$ and $\mathrm{NLO} \mathrm{W}^{+} \mathrm{W}^{-} \mathrm{b} \overline{\mathrm{b}}$ cross sections in the exclusive bins with $N_{\mathrm{b}}=0($ left $)$ and $N_{\mathrm{b}}=1$ (right) b-jets versus the b-jet- $p_{\mathrm{T}}$ threshold. Same conventions as in Fig. 4

ically disfavoured at large $p_{\mathrm{T}, \mathrm{jet}}^{\mathrm{thr}}$, while the relative importance of NLO jet emission and FtW effects increases quite dramatically.
Analogous results for exclusive bins with $N_{\mathrm{b}}=0$ and $N_{\mathrm{b}}=1 \mathrm{~b}$-jets are displayed in Fig. 5 . In this case the reduced sensitivity of b-jet bins to NLO real emission is clearly 

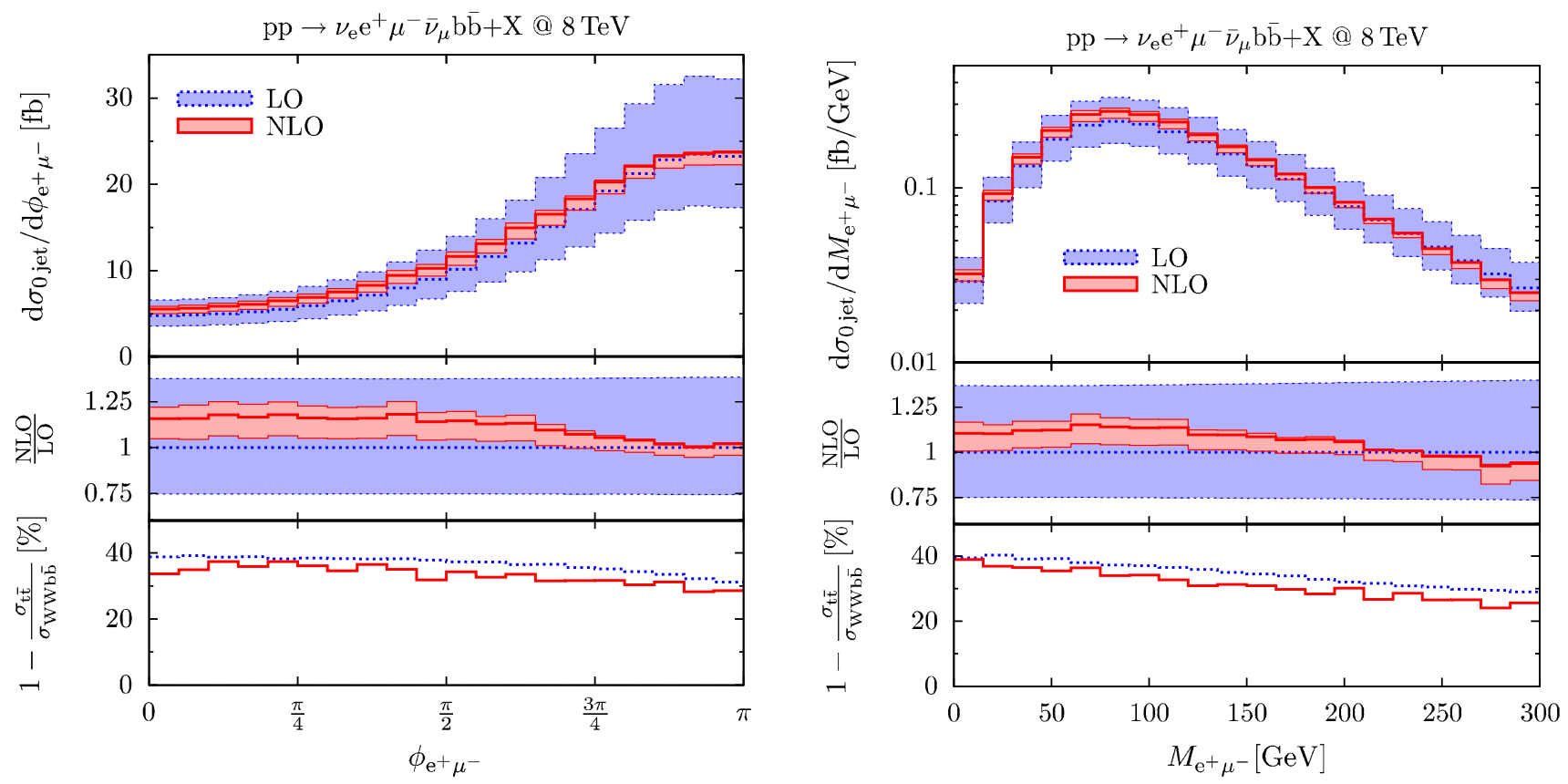

Fig. 6 Differential distributions in the 0-jet bin: azimuthal-angle separation (left) and invariant mass (right) of the two charged leptons. Same conventions as in Fig. 4

reflected in the much better stability of the $K$-factor with respect to variations of $p_{\mathrm{T}, \mathrm{bjet}}^{\text {thr }}$. Similarly as for jet bins, $\mathrm{FtW}$ corrections are strongly enhanced at small $p_{\mathrm{T}}$. This effect can be attributed to the single-top Wt channels, and the inclusion of $\mathrm{t} \overline{\mathrm{t}}-\mathrm{Wt}$ interferences, as in the present $\mathrm{W}^{+} \mathrm{W}^{-} \mathrm{b} \overline{\mathrm{b}}$ calculation, is clearly advisable in this regime.

Finally, in Fig. 6 we show distributions in the azimuthalangle-separation and in the invariant mass of charged leptons in the 0 -jet bin. These observables play a key role for the measurement of the $\mathrm{H} \rightarrow \mathrm{W}^{+} \mathrm{W}^{-}$signal at the LHC, and the accurate modelling of top backgrounds is very important for the experimental analyses. In this context, Fig. 6 shows that $\mathrm{NLO}$ and $\mathrm{FtW}$ effects are quite significant. In particular, the impact of $\mathrm{FtW}$ contributions reaches up to $40 \%$. Shape distortions due to the kinematic dependence of FtW and NLO contributions are at the $10 \%$ level, and scale variations do not exceed $10 \%$ at NLO. The fact that FtW corrections are fairly stable with respect to NLO corrections provides further evidence of the stability of the perturbative description.

\section{Summary and conclusions}

We have presented a complete NLO simulation of $\mathrm{W}^{+} \mathrm{W}^{-} \mathrm{b} \overline{\mathrm{b}}$ production at the LHC, including $\mathrm{W}$-boson decays in the opposite-flavour di-lepton channel, finite $\mathrm{W}$ - and top-width effects, and massive b-quarks in $4 \mathrm{~F}$ scheme. The finite $\mathrm{b}$ quark mass acts as a regulator of collinear singularities and allows one to describe the full b-quark phase space, including single-top contributions that arise from initial-state $g \rightarrow b \bar{b}$ splittings followed by gb $\rightarrow$ Wt scattering. This yields a gauge-invariant description of top-pair, single-top, and nonresonant $\mathrm{W}^{+} \mathrm{W}^{-} \mathrm{b} \bar{b}$ production including all interferences at NLO QCD. We introduced a dynamical scale choice aimed at an improved perturbative stability of initial-state $g \rightarrow b \bar{b}$ splittings in single-top contributions. Using this scale, the $\mathrm{NLO} \mathrm{W}^{+} \mathrm{W}^{-} \mathrm{b} \overline{\mathrm{b}}$ cross section in bins with 0,1 and 2 jets features NLO scale uncertainties at the 10-15\% level. The more conventional choice $\mu_{0}=m_{\mathrm{t}}$ yields similarly small NLO uncertainties in jet bins. While providing further evidence of the good convergence of the perturbative expansion, this means that a sophisticated dynamical scale is unnecessary for the rather inclusive observables considered in this letter. However, such a dynamical scale might become important for more exclusive observables, like jet- $p_{\mathrm{T}}$ distributions.

Finite-top-width corrections mainly originate from singletop and off-shell $\mathrm{tt}_{\mathrm{t}}$ contributions. They represent $6 \%$ of the integrated cross section and are strongly sensitive to the jet multiplicity. In the 2-jet bin they are as small as $2 \%$, while in the 1- and 0-jet bins they reach the 16 and $32 \%$ level, respectively. Also NLO corrections vary quite strongly with the jet multiplicity. Moreover, finite-top-width contributions receive quite different corrections as compared to on-shell $t \bar{t}$ production.

The non-trivial interplay of NLO and finite-width effects is especially relevant for the 0 - and 1 -jet bins. It plays an important role for the accurate description of associated $\mathrm{Wt}$ production, as well as for top backgrounds to $\mathrm{H} \rightarrow \mathrm{W}^{+} \mathrm{W}^{-}$ and to other searches based on leptons, large missing energy and jet vetoes. All employed tools are fully automated and 
can easily be exploited to extend the present results to the likeflavour di-lepton channel or to simulate any other StandardModel process at NLO QCD.

Acknowledgments We thank A. Denner, S. Dittmaier and L. Hofer for providing us with the one-loop tensor-integral library COLLIER. We are grateful to S. Höche and F. Siegert for SHERPA technical support. Our research is funded by the SNSF and supported, in part, by the European Commission through the network PITN-GA-2010-264564 (LHCPhenoNet).

Open Access This article is distributed under the terms of the Creative Commons Attribution License which permits any use, distribution, and reproduction in any medium, provided the original author(s) and the source are credited.

Funded by $\operatorname{SCOAP}^{3}$ / License Version CC BY 4.0.

\section{References}

1. C.D. White, S. Frixione, E. Laenen, F. Maltoni, JHEP 0911, 074 (2009) (0908.0631)

2. N. Kauer, D. Zeppenfeld, Phys. Rev. D 65, 014021 (2002). hep-ph/ 0107181

3. A. Denner et al., Nucl. Phys. B 724, 247 (2005). hep-ph/0505042

4. A. Denner et al., Phys. Rev. Lett. 106, 052001 (2011) (1012.3975)
5. A. Denner et al., JHEP 1210, 110 (2012) (1207.5018)

6. G. Bevilacqua et al., JHEP 1102, 083 (2011) (1012.4230)

7. R. Frederix (2013) (1311.4893)

8. F. Cascioli, P. Maierhoefer, S. Pozzorini, Phys. Rev. Lett. 108, 111601 (2012) (1111.5206)

9. A. Denner, D. Dittmaier, L. Hofer (in preparation)

10. A. Denner, S. Dittmaier, Nucl. Phys. B 658, 175 (2003)

11. A. Denner, S. Dittmaier, Nucl. Phys. B 734, 62 (2006)

12. A. Denner, S. Dittmaier, Nucl. Phys. B 844, 199 (2011)

13. F. Cascioli et al. (2013) (1309.0500)

14. F. Cascioli et al. (2013) (1309.5912)

15. A fully automated C++ Monte-Carlo generator for NLO QCD by S. Kallweit. Under development

16. S. Catani, M. Seymour, Nucl. Phys. B 485, 291 (1997). hep-ph/ 9605323

17. S. Catani et al., Nucl. Phys. B 627, 189 (2002). hep-ph/0201036

18. R. Kleiss, R. Pittau, Comput. Phys. Commun. 83, 141 (1994)

19. M. Grazzini, S. Kallweit, D. Rathlev, A. Torre (2013) (1309.7000)

20. T. Gleisberg, F. Krauss, Eur. Phys. J. C53, 501 (2008) (0709.2881)

21. T. Gleisberg et al., JHEP 0902, 007 (2009) (0811.4622)

22. F. Krauss, R. Kuhn, G. Soff, JHEP 0202, 044 (2002). hep-ph/ 0109036

23. M. Jezabek, J.H. Kuhn, Nucl. Phys. B 314, 1 (1989)

24. R.D. Ball et al., Nucl. Phys. B 867, 244 (2013) (1207.1303)

25. M. Cacciari, G.P. Salam, G. Soyez, JHEP 0804, 063 (2008) (0802. 1189) 